\title{
Sarcoma granulocítico hepático: una presentación inusual
}

\author{
$M^{a}$ A. ALAMA ZARAGOZA, A. ROBLES INIESTA, I. ROCA ADELANTADO, \\ M.A. SALES MAICAS ${ }^{1}$, A. FERRÁNDEZ IZQUIERDO ${ }^{2}$, P. ROMÁN SÁNCHEZ \\ Servicio de Medicina Interna y ${ }^{\prime}$ Anatomía Patológica. Hospital General de Requena. \\ SVS. Requena. Valencia \\ ${ }^{2}$ Departamento de Anatomía Patológica. Hospital Clínico Universitario de Valencia. SVS. \\ Valencia
}

\author{
HEPATIC GRANULOCYTIC SARCOMA: AN UNUSSUAL \\ PRESENTATION
}

\section{RESUMEN}

El sarcoma granulocítico es un tumor extramedular, poco frecuente, constituido por precursores inmaduros mieloides. Puede aparecer en ausencia de enfermedad hematológica o con anterioridad a la misma, aunque lo más usual es que se asocie a una leucemia mieloide aguda o a otro proceso mieloproliferativo, o bien que se manifieste en el curso de un síndrome mielodisplásico.

Nuestro paciente, varón de 81 años de edad, presentaba un cuadro de ictericia mucocutánea franca, aguda, con un patrón analítico de colestasis, unas técnicas de imagen negativas, lo cual nos indicaba su origen intrahepático, marcadores tumorales negativos, serología negativa. La biopsia hepática, macroscópicamente de coloración verdosa, mostraba una infiltración difusa de los sinusoides hepáticos por celularidad indiferenciada, con positividad para mieloperoxidasa en el estudio inmunohistoquímico. El paciente no presentaba afectación de sangre periférica (ninguna de las tres series) y falleció por un fallo hepatorrenal agudo a los 8 días de su ingreso.

PALABRAS CLAVE: Sarcoma granulocítico (Cloroma). Leucemia mieloide aguda. Ictericia. Estudio inmunohistoquímico. Tumor mieloide extramedular.

\begin{abstract}
A case of granulocytic sarcoma (chloroma) of hepatic localization is presented. It is a extramedullary strange tumour, composed of immature precursors of myeloid cells. Clinically it can show, before, during or after a acute myeloid leukemia, chronic myeloproliferative disorders or myelodysplastic syndromes.

Our patient, 81 year-old male, presented a process of important acu te jaundice, with negative image thecnics, what indicated us the intrahe patic origin, negative tumorals markers, negative serology and hepatic biopsy (the piece of greenish coloration is described) what showed a hepatic sinusoides diffuse infiltration by indifferentation cellularity, with study immuno-histochemical that was positive for the myeloperoxydase, giving a diagnose compatible with hepatic infiltration for acute myeloid leukemia.

The patient doesn't present affectation of peripheral blood, and he died for acute hepatic and renal failure after 8 days of entrance.
\end{abstract}

KEY WORDS: Granulocytic sarcoma (chloroma). Acute myeloid leuke mia. Jaundice. Study immuno-histochemical. Extra-medullary myeloid tumour.

Alama Zaragoza $M^{a}$ A, Robles Iniesta A, Roca Adelantado I, Sales Maicas MáA, Ferrández Izquierdo A. Roman Sánchez P. Sarcoma granulo cítico hepático: una presentación inusual. An Med Interna (Madrid) 2003; 20: 141-144.

\section{INTRODUCCIÓN}

El sarcoma granulocítico o cloroma, es un tumor mieloide extramedular, poco frecuente, que se constituye de precursores inmaduros de células mieloides $(1-3,7,9,18,19)$. Puede aparecer en cualquier momento de la vida, incluso en la edad pediátrica $(12,23)$.

Clínicamente puede manifestarse antes, durante o después de una leucemia mieloide aguda, proceso mieloproliferativo o síndrome mielodisplásico (1-3,5,6,9). Únicamente un porcentaje muy bajo de pacientes, no desarrollan afectación medular (15).
El cloroma puede aparecer en cualquier órgano o tejido $(17,19,21)$. Sus localizaciones más frecuentes son en hueso, partes blandas, ganglio y piel (21). Menos frecuente es su presentación en ovario (4), pulmón, útero, próstata (11) y tracto gastrointestinal (1-3,5,9). De su lugar de asentamiento dependerá su expresión clínica.

El diagnóstico precoz es fundamental, aunque difícil, sobretodo en aquellos casos en los que no se observa afectación en sangre periférica, y por tanto, no existe sospecha diagnóstica (9). Se establece fundamentalmente por punción aspiración con aguja fina $(3,6,8,16)$ (suelen ser piezas de coloración verdosa (14), de ahí el nombre de cloroma), con

Trabajo aceptado: 20 de febrero de 2002

Correspondencia: M. ${ }^{\text {a }}$ Amparo Alama Zaragoza. Servicio de Medicina Interna. Hospital General de Requena. Servicio Valenciano de Salud. Paraje Casablanca, s/n. 46340 Requena (Valencia). e-mail: robles-iniesta@mixmail.com 
posterior estudio anatomopatológico e inmunohistoquímico $(7,9,11,13,15,24)$. Histológicamente, la morfología del sarcoma granulicítico es variable, así se pueden encontrar tumores bien diferenciados, constituidos por elementos celulares que semejan representar los distintos estadios de diferenciación mieloide con células similares a mielocitos eosinófilos fácilmente reconocibles, o por el contrario, neoplasias de formas blásticas sin diferenciación granulocítica aparente. La inclusión de marcadores de línea mieloide en el estudio inmunohistoquímico de estos tumores indiferenciados es primordial para su diagnóstico correcto.

El tratamiento de este proceso pasa por la cirugía, radioterapia y quimioterapia $(11,19)$ con resultados variables, en general poco esperanzadores (12).

\section{CASO APORTADO}

Paciente varón, de 81 años de edad que ingresa por cuadro de ictericia de reciente aparición, sin prurito, con coluria e hipocolia, asociando, semanas antes, incremento de edemas en miembros inferiores y del perímetro abdominal.

Como antecedentes personales refería: hábito enólico importante y persistente hasta su ingreso, exfumador desde hace 10 años, EPOC de grado moderado, DM tipo 2 en tratamiento con glimepirida desde 15 días antes de su ingreso, obesidad por hiperalimentación, hernia hiatal, Fiebre de Malta hacía años. Su tratamiento actual era: glimepirida, espironolactona, fluticasona, bromuro de ipatropio, omeprazol y un venotónico. El paciente negaba la ingesta de paracetamol y benzodiacepinas.

En la exploración física: paciente consciente, orientado, normotenso, normohidratado, eupneico, afebril, obeso y con ictericia mucocutanea franca. Auscultación cardiopulmonar normal, abdomen globuloso con dudas sobre la existencia de ascitis, miembros inferiores con edemas hasta la rodilla de aspecto crónico, alguna telangiectasia aislada, lengua saburral, Dupuytren leve bilateral y un nivel cognitivo adecuado con asterixis leve.

Exploraciones complementarias: Analítica: Hemograma: $\mathrm{Hb}$ $15.9 \mathrm{~g} / \mathrm{dl}, \mathrm{Htcr} 47.8 \%$, VCM $104 \mathrm{fl}$, Leucocitos 13.900 miles /ul con fórmula y recuento normales, Plaquetas 135.000 miles/ ul, VSG 24 $\mathrm{mm}$ en la $1^{\mathrm{a}}$ hora. Coagulación: TTPA $47 \mathrm{seg}$, I. Quick 43\%, fibrinogeno $271 \mathrm{mg} / \mathrm{dl}$. Bioquímica sérica: bilirrubina total 24,9 mg/dl, bilirrubina directa $18,5 \mathrm{mg} / \mathrm{dl}$, bilirrubina indirecta $7,8 \mathrm{mg} / \mathrm{dl}$, GOT 83 UI/L, GPT 27 UI/L, GGT $140 \mathrm{UI} / \mathrm{L}, \mathrm{LDH} 1041 \mathrm{UI} / \mathrm{L}$, fosfatasa alcalina $409 \mathrm{UI} / \mathrm{L}$, proteínas totales 5,42 g/dl, albúmina 2,33 g/dl, glucosa $88 \mathrm{mg} / \mathrm{dl}$, urea $42 \mathrm{mg} / \mathrm{dl}$, creatinina $1,01 \mathrm{mg} / \mathrm{dl}, \mathrm{Na} 137 \mathrm{mmol} / \mathrm{L}, \mathrm{K}$ $5,07 \mathrm{mmol} / \mathrm{L}$, Ca $9,3 \mathrm{mg} / \mathrm{dl}$, fósforo 3,5 mg/dl, colesterol $67 \mathrm{mg} / \mathrm{dl}$, triglicéridos $101 \mathrm{mg} / \mathrm{dl}$, CPK $40 \mathrm{UI} / \mathrm{L}$, amilasa $26 \mathrm{UI} / \mathrm{L}$, fe sérico 131 $\mathrm{mcg} / \mathrm{dl}$, transferrina $92 \mathrm{mg} / \mathrm{dl}$, ferritina $716 \mathrm{ng} / \mathrm{ml}$, capacidad total de fijación del hierro 117, índice de saturación de transferrina 142\%, marcadores tumorales (PSA, CEA, AFP) negativos, Ac antimitocondriales negativos, serología para Brucella, VHA, VHB, VHC negativa.

Proteinograma: moderada hipoalbuminemia con hipergammaglobulinemia policlonal. Bioquímica de orina: anormales y sedimento sin alteraciones. Gasometría arterial $\left(\sin \mathrm{O}_{2}\right)$ : $\mathrm{pH} 7,44, \mathrm{pO}_{2} 62,5$ $\mathrm{mmHg}, \mathrm{pCO}_{2} 37,3 \mathrm{mmHg}$, bicarbonato $25 \mathrm{mmol} / \mathrm{L}$, saturación de $\mathrm{O}_{2}$ 92\%. Rx tórax: signos de EPOC sin condensaciones ni derrame. $R x$ abdomen: sin alteraciones significativas. ECG: ritmo sinusal normal. Ecografía abdominal: hígado homogéneo, sin nodularidad, pero con contornos redondeados y lobulados sugestivo de hepatopatía crónica. No se objetiva esplenomegalia ni ascitis, vena porta, vesícula biliar y vía biliar tanto intra como la extrahepática de características normales. TAC abdominal: estudio realizado tras administrar contraste intravenoso que muestra hígado homogéneo sin nodularidad, con contornos redondeados y lobulados sugestivos de hepatopatía cróni- ca. Resto sin alteraciones. Ecocardiografía: Cavidades izquierdas normales, ligera dilatación de cavidades derechas con leve insuficiencia tricuspídea y moderada hipertensión pulmonar. Estudio histopatológico: Se obtuvo biopsia cilíndrica hepática de coloración verdosa, que fue fijada en formol e incluida en parafina, según técnica habitual. Realizamos las tinciones convencionales sobre secciones de 4 micras, que pusieron en evidencia una infiltración neoplásica hepática masiva (Fig. 1). Se trataba de células monomorfas de citoplasma claro, PAS negativas, contorno poligonal y núcleo redondeado con cromatina granular. Esta invasión tumoral se localizaba en los sinusoides hepáticos, donde las células malignas constituían agregados a modo de nidos y cordones sin estroma de sostén. Ocasionalmente parecían agruparse entorno a un espacio luminal central (Fig. 2). Las trabéculas de hepatocitos se encontraban comprimidas por las células tumorales. En los hepatocitos se observaba acúmulo de pigmento bilirrubina intracitoplasmático y con frecuencia, imágenes de muerte celular. Estudio inmunohistoquímico: únicamente mostró positividad frente a mieloperoxidasa en la población neoplásica siendo negativo para: CD 20, CD 3, CD 68, citoqueratinas y cromogranina. Así pues, se trataba de una infiltración hepática por precursores inmaduros mieloides, que en ausencia de enfermedad medular, posibilita el diagnóstico de: tumor mieloide extramedular/sarcoma gra nulocítico.

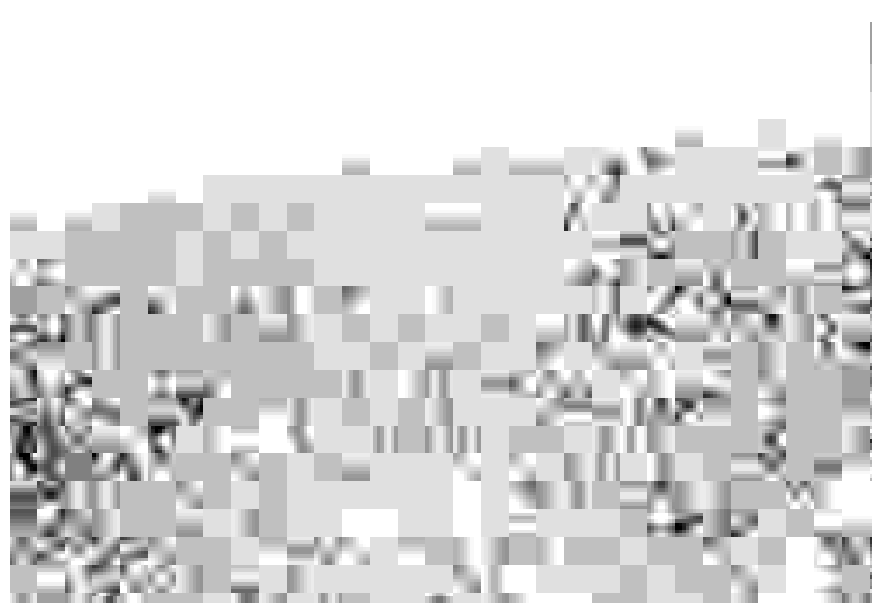

Fig. 1. Infiltración masiva del tejido hepático por nidos de células de citoplasma pálido (original, HE x 50).

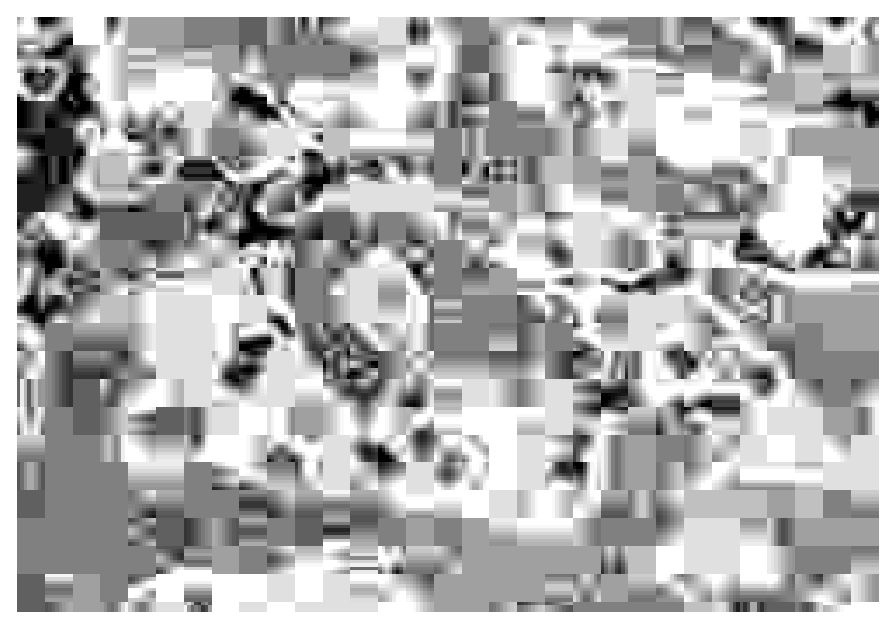

Fig 2. Celularidad monomorfa, de contorno poligonal, delimitando ocasionalmente espacios pseudoacinares en los sinusoides hepáticos (original, HE x 400). 
Evolución: el paciente, durante su estancia hospitalaria, sufrió un deterioro de su estado general, con importante disminución del volumen de diuresis y afectación neurológica a pesar del tratamiento médico aplicado, presentando la siguiente analítica previa a su fallecimiento: urea $234 \mathrm{mg} / \mathrm{dl}$, creatinina 5,18 mg/dl, colesterol $36 \mathrm{mg} / \mathrm{dl}$, Ac. úrico $10.7 \mathrm{mg} / \mathrm{dl}$, fosfatasas alcalinas $341 \mathrm{UI} / \mathrm{L}, \mathrm{Na} 140 \mathrm{mmol} / \mathrm{L}$, $\mathrm{K} 7,43 \mathrm{mmol} / \mathrm{L}$, bilirrubina total $38,03 \mathrm{mg} / \mathrm{dl}$, bilirrubina directa 25,09 mg/dl, e indirecta 12,94 mg/dl, GOT $237 \mathrm{UI} / \mathrm{L}$, GPT $75 \mathrm{UI} / \mathrm{L}$, GGP 125UI/L, I. Quick 19\%, TTPA 63,3 seg, pH 7,21, bicarbonato 9 $\mathrm{mmol} / \mathrm{L}$.

\section{DISCUSIÓN}

La existencia de un sarcoma granulocítico es poco frecuente $(1,19)$, pero su afectación hepática se puede considerar una rareza (20), siendo más frecuente su aparición en otras localizaciones (1) como son: hueso, partes blandas, piel y ganglios (21). Se trata de un tumor constituido por precursores inmaduros de células mieloides y puede aparecer antes, durante o después de una leucemia mieloide aguda, proceso mieloproliferativo o síndrome mielodisplásico $(1-3,7,9,18,19)$. El diagnóstico precoz de esta entidad es necesario y difícil al mismo tiempo $(9,22)$, siendo la prueba diagnóstica de elección, la punción aspiración con aguja fina $(3,6,8,16)$.

En el caso que nos ocupa, se presenta un paciente de edad avanzada, con un patrón analítico de colestasis que podemos catalogar de intrahepática, dada la ausencia de hallazgos en las técnicas de imagen que pudieran indicar alguna causa tanto benigna como maligna de afectación extrahepática y con una alteración del metabolismo férrico justificado en el contexto de una hepatopatía crónica. Dentro de las múltiples causas de colestasis intrahepática, se tuvieron en cuenta las siguientes como más probables, tras realizar el diagnóstico diferencial: hepatitis alcohólica por el importante hábito enólico del paciente (pese a no existir GOT/GPT >2, ni elevación importante de la GGP), hepatitis tóxica causada por la glimepirida (como RAM de este fármaco esta descrita la aparición de ictericias y en casos más graves fallo hepático fulminante), hepatocarcinoma difuso e infiltración hepática por otras patologías como linfomas, leucemias, etc, aunque la ausencia de positividad para los marcadores tumorales nos hacía dudar de que se tratara de un hepatocarcinoma difuso. De todos modos para poder establecer un diagnóstico definitivo, era imprescindible, la realización de una biopsia hepática y su posterior estudio anatomopatológico e inmunohistoquímico.
El estudio de la biopsia hepática con técnicas de rutina, evidenció una infiltración hepática difusa a nivel sinusoidal por celularidad maligna indiferenciada. En nuestro caso la inmunohistoquímica fue de gran ayuda, ya que era un tumor de difícil reconocimiento histológico en un paciente donde no existía sospecha clínica. Por ello proponemos, junto a otros autores $(15,24)$, incluir en el estudio inmunohistoquímico de tumores indiferenciados, marcadores de línea mieloide, de alta sensibilidad y especificidad como son la mieloperoxidasa y/o la lisozima. Queremos destacar que, en la literatura, hemos encontrado algunos casos similares histológicamente al que nos ocupa. En todos ellos, las células neoplásicas constituyen un patrón inusual de infiltración en nidos y cordones, con gran cohesividad entre sus elementos celulares semejando carcinomas $(24,26)$. Se ha evidenciado la expresión de moléculas de adhesión y la formación de uniones estrechas en uno de estos casos con afectación cutánea, postulándose que sería un entorno propicio y diferente al medular, donde estas células son redondas, el que estimularía su desarrollo (27).

En general, la evolución de esta patología es poco favorable (12) y va a depender, de un diagnóstico precoz, para poder aplicar el tratamiento adecuado, de la buena respuesta al mismo, del lugar de localización del proceso (19) y de la aparición de recidivas posteriores o de una leucemia mieloide aguda (19) en el caso de que esta no estuviera ya presente.

El tratamiento engloba la cirugía, cuando el proceso es susceptible de resección, seguido de quimioterapia y radioterapia $(11,19)$, con resultados variables y en general poco satisfactorios (12). Están descritos casos de supervivencia de unos 6 meses en un cloroma pulmonar, pese a quimioterapia (14), así como también se encuentran en la literatura, casos de aparición de una leucemia mieloide aguda, tras 6-7 meses de la resección quirúrgica de un cloroma en partes blandas (16) y 17 meses después del tratamiento con quimioterapia de una masa retro-orbital (10).

En nuestro caso tenemos un paciente que desarrolló, tras 8 días de ingreso, un fallo hepatorrenal agudo que condicionó su fallecimiento, en el contexto de un cuadro de colestasis intrahepática, con técnicas de imagen y marcadores tumorales negativos, sin afectación de ninguna de las tres series en sangre periférica y con un estudio inmunohistoquímico de la biopsia hepática, que dió un diagnóstico de infiltración hepática por una leucemia mieloide aguda, con lo cual, nos hallamos ante la rara entidad denominada sarcoma granulocitico de localización hepática.

\section{Bibliografía}

1. Saleh HA, Khatib G. Fatal gastrointestinal bleeding as the primary manifestation of granulocytic sarcoma in a patient with myelodysblastic síndrome. J Fla Med Assoc 1997; 84 (2):111-4.

2. Ravandi-Kashani F, Estey E, Cortes J, Medeiros LJ, Giles FJ. Granulocytic sarcoma of the pancreas: a report of two cases and literature review. Clin Lab Haematol 1999; 21 (3): 219-24.

3. Paydas S, Hazar B, Sahin B, Gonlusen G. Granulocytic sarcoma as the cause of giant abdominal mass: diagnosis by fine needle aspiration and review of the literature. Leuk Res 2000; 24 (3): 267-9.

4. Jung SE, Chun KA, Park SH, Lee EJ. MR findings in ovarian granulocytic sarcoma. Br J Radiol 1999; 72 (855): 301-3.

5. Takeh H, Farran M, Debaize JP. Granulocytic sarcoma (chloroma) of the small intestine. Acta Chir Belg 1999; 99 (2): 78-81.

6. Bangerter M, Hildebrand A, Waidmann O, Griesshammer M. Diagnosis of granulocytic sarcoma by fine needle aspiration cytology. Acta Haematol 2000; 103 (2): 102-8.

7. Colle I; Lacor P; Peeters P; Jochmans K; De Raeve H; Van Camp B. Granulocytic sarcoma (chloroma): a report of two cases. Acta Clin Belg 1996; 51 (2): 106-10.

8. Suh YK, Shin HJ. Fine-needle aspiration biopsy of granulocytic sarcoma: a clinicopathologic study of 27 cases. Cancer 2000; 90 (6): 364-72.

9. Brugo EA, Marshall RB, Riberi AM, Pautasso OE. Preleukemic granulocytic sarcomas of the gastrointestinal tract. Report of two cases. Am J Clin Pathol 1977; 68 (5): 616-21. 
10. Van Veen S, Kluin PM, de Keizer RJ, Kluin-Nelemans HC. Granulocytic sarcoma (chloroma). Presentation of an unusual case. Am J Pathol 1991; 95 (4): 567-71.

11. Quien ET, Wallach B, Sandhaus L, Kidd P, Strair R, Saidi P. Primary extramedullary leukemia of the prostate: case report and review of the literature. Am J Hematol 1996; 53 (4): 267-71.

12. Capdeville R, Bertrand Y, Manel AM, Charrin C, Souillet G, Philippe N Granulocityc sarcoma (chloroma): rare extramedullary tumors associated with acute non-lymphoblastic leukemia. Pediatrie 1990; 45 (4): 245-50.

13. Muller S, Sangster G, Crocker J, Nar P, Burnett D, Brown G, Leyland MJ. An immunohistochemical and clinicopathological study of granulocytic sarcoma (chloroma). Hematol Oncol 1986; 4 (2): 101-12.

14. Le Charpentier Y, Mathiot C, Varet B, Lacombe MJ, Carlioz A Vacher-Lavenu MC, et al. Aleukemic chloroma. Review of literature and diagnostic problems: 1 case. Ann Anat Pathol (Paris) 1978; 23 (3): 217-32.

15. Menasce LP, Banerjee SS, Beckett E, Harris M. Extra-medullary myeloid tumour ( granulocityc sarcoma) is often misdiagnosed: a study of 26 cases. Histopathology 1999; 34 (5): 391-8.

16. Kumar PV. Soft tissue chloroma diagnosed by fine needle aspiration cytology. A case report. Acta Cytol 1994; 38 (1): 83-6.

17. Mathiot C, Le Charpentier Y, Sol C, Varet B, Levy JP. Multifocal Chloroma (granulocytic sarcoma), without marrow involvement. One case. Nouv Presse Med 1978; 7 (28): 2461-3.

18. Deptala A, Paluszewska M, Maryniak R, Rymkiewicz K. Chloroma a rare symptom of acute leukemia. Pol Tyg Lek 1994; 49 (12-13): 291-3.
19. Mwanda WO, Rajab JA.. Granulocytic sarcoma: report of three cases. East Afr Med J 1999; 76 (10): 594-6.

20. Matsueda K, Yamamoto H, Dio I. An autopsy case of granulocytic sarcoma or the porta hepatis causing obstructive jaundice. J Gastroenterol 1998; 33 (3): 428-33.

21. Robaglia A, Horschowski N, Sainty D, Xerri L, Guitard AM, Gastaut JA, Dhiver C, Seite R. Multicentric bone chloroma disclosed by pleural cytology. Arch Anat Cytol Pathol 1990; 38 (1-2): 19-21.

22. Buxo J, Bandal JM, Perez de Olaguer J, Majo J. Granulocytic sarcoma: unusual form of presentation for acute nonlymphoblastic leukemia. Sangre (Bar) 1997; 42 (1): 73-5.

23. Yonemitsu T, Fujiwara S, Yoshimoto T, Ohwada K, Minegishi M. A case of granulocytic sarcoma (cloroma) preceding acute leukemia. No Shinkei Geka 1986; 14 (1): 67-72.

24. Chang Ch-Ch, Eshoa C, Kampalath B, Shidham VB, Perkins S Immunophenotypic profile of myeloid cells in granulocytic sarcoma by immunohistochemistry. Am J Clin Pathol 2000; 114: 807 11.

25. Oliva E, Ferry JA, Young RH, Prat J, Srigley JR, Scully RE. Granulocytic sarcoma of the female genital tract: a clinicopathologic study of 11 cases. Am J Surg Pathol 1997; 21: 1156-65.

26. Hamoudi WH, Medeiros LJ, Townsend G, Glassman AB, BuesoRamos CE. Arch Pathol Lab Med 2000; 124: 315-8.

27. Asano T, Fukuda Y, Katsube Y, et al. Infantile acute monocytic leukemia with tumor formation in the skyn expressing adhesion molecules as seen electron microscopy. Leuk Lynphoma 1996; 23: 173-9. 